\title{
Remitting seronegative symmetrical synovitis with pitting oedema (RS3PE) syndrome: a prospective follow up and magnetic resonance imaging study
}

Fabrizio Cantini, Carlo Salvarani, Ignazio Olivieri, Libero Barozzi, Luigi Macchioni, Laura Niccoli, Angela Padula, Pietro Pavlica, Luigi Boiardi

\begin{abstract}
Objective-To determine the clinical characteristics of patients with "pure" remitting seronegative symmetrical synovitis with pitting oedema (RS3PE) syndrome, and to investigate its relation with polymyalgia rheumatica (PMR). Magnetic resonance imaging (MRI) was used to describe the anatomical structures affected by inflammation in pure RS3PE syndrome.

Methods-A prospective follow up study of 23 consecutive patients with pure RS3PE syndrome and 177 consecutive patients with PMR diagnosed over a five year period in two Italian secondary referral centres of rheumatology. Hands or feet MRI, or both, was performed at diagnosis in 7 of 23 patients.
\end{abstract}

Results-At inspection evidence of hand and/or foot tenosynovitis was present in all the 23 patients with pure RS3PE syndrome. Twenty one (12\%) patients with PMR associated distal extremity swelling with pitting oedema. No significant differences in the sex, age at onset of disease, acute phase reactant values at diagnosis, frequency of peripheral synovitis and carpal tunnel syndrome and frequency of HLA-B7 antigen were present between patients with pure RS3PE and PMR. In both conditions no patient under 50 was observed, the disease frequency increased significantly with age and the highest frequency was present in the age group 70-79 years. Clinical symptoms for both conditions responded promptly to corticosteroids and no patient developed rheumatoid arthritis during the follow up. However, the patients with pure RS3PE syndrome were characterised by shorter duration of treatment, lower cumulative corticosteroid dose and lower frequency of systemic signs/symptoms and relapse/recurrence. Hands and feet MRI showed evidence of tenosynovitis in five patients and joint synovitis in three patients.

Conclusion-The similarities of demographic, clinical, and MRI findings between RS3PE syndrome and PMR and the concurrence of the two syndromes suggest that these conditions may be part of the same disease and that the diagnostic labels of PMR and RS3PE syndrome may not indicate a real difference. The presence of distal oedema seems to indicate a better prognosis.

(Ann Rheum Dis 1999;58:230-236)
In 1985 and 1990 McCarty et al described 23 patients with remitting symmetrical synovitis of upper limbs associated with pitting oedema of the dorsum of hands. ${ }^{12}$ The acronym RS3PE (remitting, seronegative, symmetrical synovitis with pitting oedema) was used to define this syndrome. Large joint arthritis and/or pitting oedema of the dorsum of feet coexisted in some patients. All patients were negative for rheumatoid factor. A symmetrical synovitis affecting flexor tendon sheaths, wrists and joints of the hand was considered to be the prominent clinical finding. The syndrome occurred in elderly patients and it was usually associated with increased acute phase reactants. HLA-B7 was observed in $59 \%$ of the patients.

The clinical findings rapidly remitted without recurrences after a brief period of low doses of corticosteroids. However, a residual decreased motion of wrists or fingers, or both, attributable to flexion contractures was observed in 14 of 23 (61\%) of the cases. ${ }^{12}$ Additional cases of asymmetrical RS3PE syndrome were also reported. ${ }^{3}{ }^{4}$ Subsequently, the clinical findings of RS3PE have also been described in other rheumatic diseases such as polymyalgia rheumatica, ${ }^{56}$ spondylarthropathies, ${ }^{7-9}$ psoriatic arthritis, ${ }^{10}$ rheumatoid arthritis, ${ }^{11}$ acute sarcoidosis. ${ }^{12}$ Moreover, a paraneoplastic RS3PE syndrome has been recently reported. ${ }^{13-15}$ Therefore, the acceptance of RS3PE syndrome as an autonomous entity has been questioned in recent papers. ${ }^{6}{ }^{10}$ Swelling with pitting oedema may represent a clinical sign of a vigorous distal tenosynovitis common to different rheumatic conditions.

To evaluate the existence of RS3PE syndrome as a distinct syndrome and its demographic, clinical, and prognostic features, we have prospectively followed up 23 consecutive patients with this syndrome in its isolated form observed over a five year period.

Hand or foot magnetic resonance imaging (MRI), or both, was performed to investigate the anatomical structures affected by inflammation. We have also compared the demographic, clinical, and immunogenetic findings of these patients with "pure" RS3PE syndrome with those of 177 consecutive polymyalgia rheumatica (PMR) patients diagnosed over the same five year period in the same areas.

\section{Methods}

All patients with distal extremity swelling with pitting oedema seen in two Italian hospitals (Prato and Reggio Emilia) over a five year period (1992-1996) were considered suitable
Accepted for publication 7 January 1999 
Table 1 Comparisons among the demographic and clinical characteristics of the patients with PMR with and without distal pitting oedema and "pure" RS3PE syndrome

\begin{tabular}{|c|c|c|c|c|c|}
\hline & \multirow{2}{*}{$\begin{array}{l}\text { Patients with PMR } \\
\text { without distal pitting } \\
\text { oedema }(n=156) A\end{array}$} & \multirow{2}{*}{$\begin{array}{l}\text { Patients with PMR } \\
\text { and distal pitting } \\
\text { oedema }(n=21) B\end{array}$} & \multirow{2}{*}{$\begin{array}{l}\text { Patients with pure } \\
\text { RS } 3 P E \text { syndrome } \\
(n=23) C\end{array}$} & \multicolumn{2}{|l|}{ p value } \\
\hline & & & & $C v A$ & $C v B$ \\
\hline Male/female (\%) & $33 / 67$ & $43 / 57$ & $52 / 48$ & 0.06 & NS \\
\hline Age at onset of disease (y) & $70 \quad(8)$ & $75 \quad(4)$ & $74 \quad(9)$ & NS & NS \\
\hline Duration of disease before diagnosis (months) & $3.0(1.2)$ & $3.0(1.1)$ & $1.6(0.8)$ & 0.001 & 0.001 \\
\hline Duration of treatment (months) & $22(20)$ & $14 \quad(8)$ & 5 (5) & 0.001 & 0.001 \\
\hline Duration of follow up (months) & $41 \quad(23)$ & $25 \quad(16)$ & $17 \quad(15)$ & 0.001 & NS \\
\hline Peripheral arthritis $(\%)$ & 26 & 19 & 22 & NS & NS \\
\hline Carpal tunnel syndrome (\%) & 15 & 9.5 & 22 & NS & NS \\
\hline Systemic symptoms and signs (fever, anorexia, weight loss) (\%) & 59 & 57 & 9 & 0.0001 & 0.001 \\
\hline Biopsy confirmed GCA (\%) & 10 & 0 & 0 & NS & NS \\
\hline Initial prednisone dose (mg/day) & $20 \quad(12)$ & $14 \quad(4)$ & $17.5(5)$ & NS & 0.02 \\
\hline Cumulative prednisone dose (g) & $5.4(4.1)$ & $3.5(1.8)$ & $1.7(1.1)$ & 0.0001 & 0.001 \\
\hline ESR at diagnosis $(\mathrm{mm} / 1 \mathrm{st} \mathrm{h})$ & $79 \quad(25)$ & $75 \quad(29)$ & $74 \quad(30)$ & NS & NS \\
\hline $\mathrm{ESR}<30 \mathrm{~mm} / 1 \mathrm{st}$ h at diagnosis (\%) & 5 & 9 & 13 & NS & NS \\
\hline $\mathrm{CRP}$ at diagnosis $(\mathrm{mg} / \mathrm{dl})$ & $6.5(5.3)$ & $7.0(3.7)$ & $6.3(5.0)$ & NS & NS \\
\hline HLA-B7 $(\%)^{\star}$ & 9 & 17 & 15 & NS & NS \\
\hline Relapse/recurrence (\%) & 32 & 43 & 13 & 0.08 & 0.04 \\
\hline Residual contractures (\%) & 0 & 0 & 9 & 0.02 & NS \\
\hline
\end{tabular}

${ }^{\star}$ HLA-B7 was determined in 80 PMR patients without distal pitting oedema, 12 PMR patients with distal pitting oedema and 13 patients with pure RS3PE syndrome. Data shown as mean (SD).

candidates for the study. We also included patients with unilateral involvement of hands or feet, or both.

To evaluate only patients with pure RS3PE syndrome, we excluded at diagnosis and during the follow up patients satisfying Healey criteria for PMR, ${ }^{16}$ patients meeting the 1987 modified ARA criteria for rheumatoid arthritis, ${ }^{17} \mathrm{pa}-$ tients meeting the ESSG criteria for the spondylarthopathies, ${ }^{18}$ and those associating psoriasis, HLA-B27 antigen, inflammatory bowel diseases, radiological evidence of sacroiliitis or chondrocalcinosis, acute sarcoidosis and neoplasia.

Twenty three patients with pure RS3PE syndrome were identified and prospectively followed up. Temporal artery biopsy specimens were obtained only in patients with cranial signs or symptoms.

The patients were clinically assessed by the same physician at presentation, monthly for the first six months and then every three months during the follow up period.

A standardised data collection form was used at every visit to record medical information. Arthritis was defined by the presence of joint swelling and pain. Tenosynovitis was defined by the presence of swelling and tenderness along a well defined tenosynovial structure. The clinical symptoms and the physical findings for carpal tunnel syndrome (CTS) (numbness or paresthesias of the thumb, index, middle and one half of the ring finger, usually worsening in nocturnal hours associated or not with positivity of Tinel's sign or Phalen's manoeuvre, or both) were also assessed.

To assess the residual functional limitation after the resolution of the acute phase at each visit the thickening of flexor and extensor tendons (evaluated by physical examination) and the range of motion of wrists, ankles and fingers were evaluated considering as normal articular range of motion the values reported by Polley and Hunder. ${ }^{19}$

In the last seven consecutive patients the tenosynovial sheath and joint involvement during the acute phase were examined using magnetic resonance imaging (MRI). Hand MRI was performed also in five age matched healthy controls. MRI of the hands or feet, or both, was performed using a $0.5 \mathrm{~T}$ superconductive magnet system (MR Max Plus, GE Medical Systems, Milwaukee, WI). Patients were placed prone, with their arms extended above the head. Both hands, joined in the "prayer position" with a foam pad between them and immobilised with tape, were positioned in the centre of a $17 \mathrm{~cm}$ bore transmit-receive extremity coil. To study the proximal and intemediated parts of the feet, each foot, in neutral position with extended knee, was placed in the centre of the same $17 \mathrm{~cm}$ bore transmit-receive coil used for hand MRI. To avoid alterations because of overturn, the forefoot was covered in aluminium foil.

Pulse sequences included sagittal T1 weighted (460/20/4 repetition time msec/echo time msec/excitations), axial proton density (2000/25/2) and T2 weighted (2000/90/2) scans. Sagittal and axial section thickeness were $3 \mathrm{~mm}$ and $7 \mathrm{~mm}$, respectively, with an intersection gap of $1 \mathrm{~mm}$. The field of view was $20 \mathrm{~cm}$, and the matrix size $160 \times 224$ or $128 \times 192$.

Scan images were examined by two radiologists (LB and PP) who had no knowledge of the physical examination. The joint space and synovial sheaths of hand or foot tendons, or both, were evaluated for fluid collection.

At diagnosis and during the follow up the erythrocyte sedimentation rate (ESR) (Westergren method) and $C$ reactive protein (CRP) (nephelometric method, NA latex CRP kit, Behringwerke, Marburg, Germany) (normal $\leqslant$ $0.5 \mathrm{mg} / \mathrm{dl}$ ) were determined. To detect joint erosions, chondrocalcinosis or sacroiliitis roentgenographs of the hands or feet, or both, and pelvis were performed in all patients at the first visit and during the follow up.

HLA-B27 was determined in all patients. Complete class I MHC antigens were determined in 13 of 23 patients using a modification of two stage National Institute of Health complement microcytotoxicity test. ${ }^{20}$ The healthy control group consisted of 126 blood donor volunteers from the same geographical areas. 
Relapse and recurrence were considered present if articular signs or symptoms occurred in a patient receiving the treatment or after treatment ended.

The end of the disease was the date of permanent cessation of treatment without relapse or recurrence. The end point of patient follow up was the date of the last visit or the date of death.

During the same five year period we conducted a prospective follow up study to evaluate the clinical and demographic features of PMR. ${ }^{6}$ We identified 177 consecutive patients. Twenty one of $177(12 \%)$ presented at least one episode of distal extremity swelling with pitting oedema. Complete class I MHC antigens were determined in 80 of 156 PMR
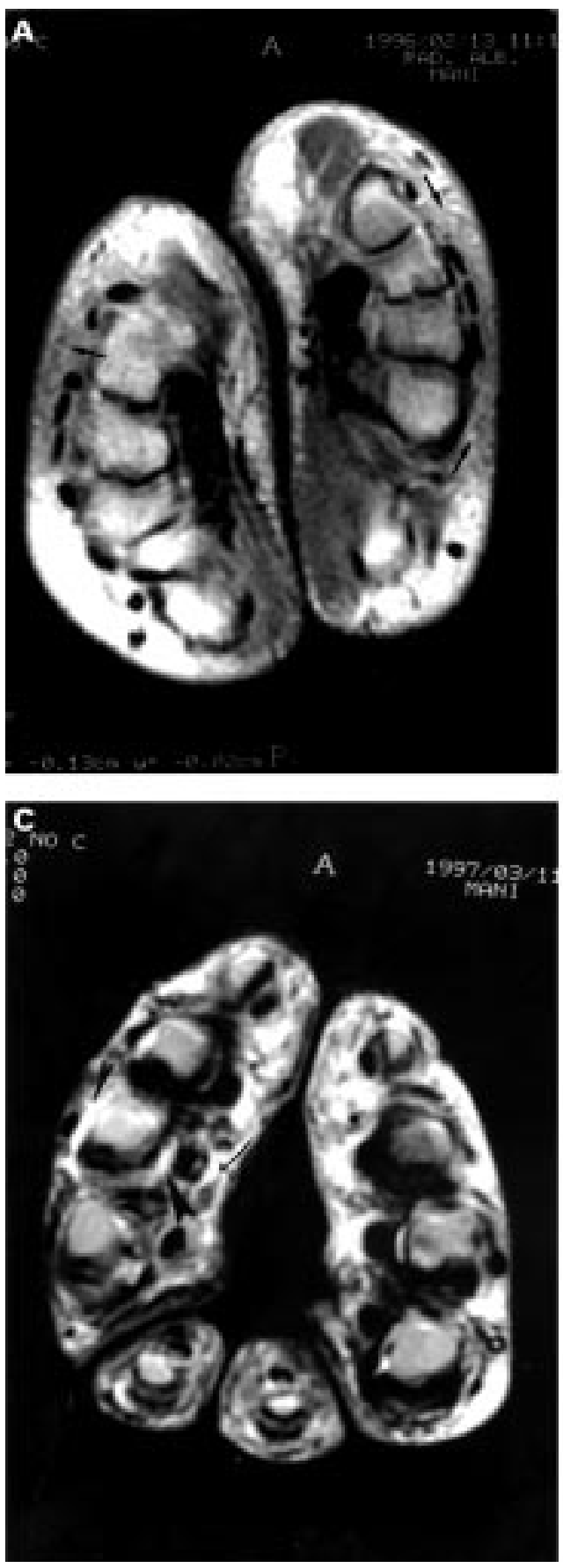

patients without distal pitting oedema and 12 of 21 PMR patients with pitting oedema. The demographic, immunogenetic, and clinical findings of the 23 patients with pure RS3PE syndrome were compared with those of 177 PMR patients with and without distal extremity swelling with pitting oedema.

Statistical analysis was done using SPSS statistical package (SPSS Inc, Chicago, Illinois). The $\chi^{2}$ and $t$ test for independent values were used.

\section{Results}

CLINICAL AND LABORATORY FINDINGS

Table 1 shows the clinical and demographic characteristics of the 23 patients with pure RS3PE syndrome. All the patients were sero-



Figure 1 MRI of the hands of RS3PE syndrome. (A) Axial proton density section through the midpoint of the palm shows subcutaneous oedema in hand dorsum (black arrows). (B) Axial T2 weighted section through the midpoint of the palm shows fluid collection in the extensor synovial sheaths of the right hand (open arrows). (C) Axial T2 weighted section through the

metacarpophalangeal joints shows fuid collection in the flexor synovial sheaths of the third digit of right hand

(curved arrow) and joint effusion of the corresponding joint (large and small arrowheads). 

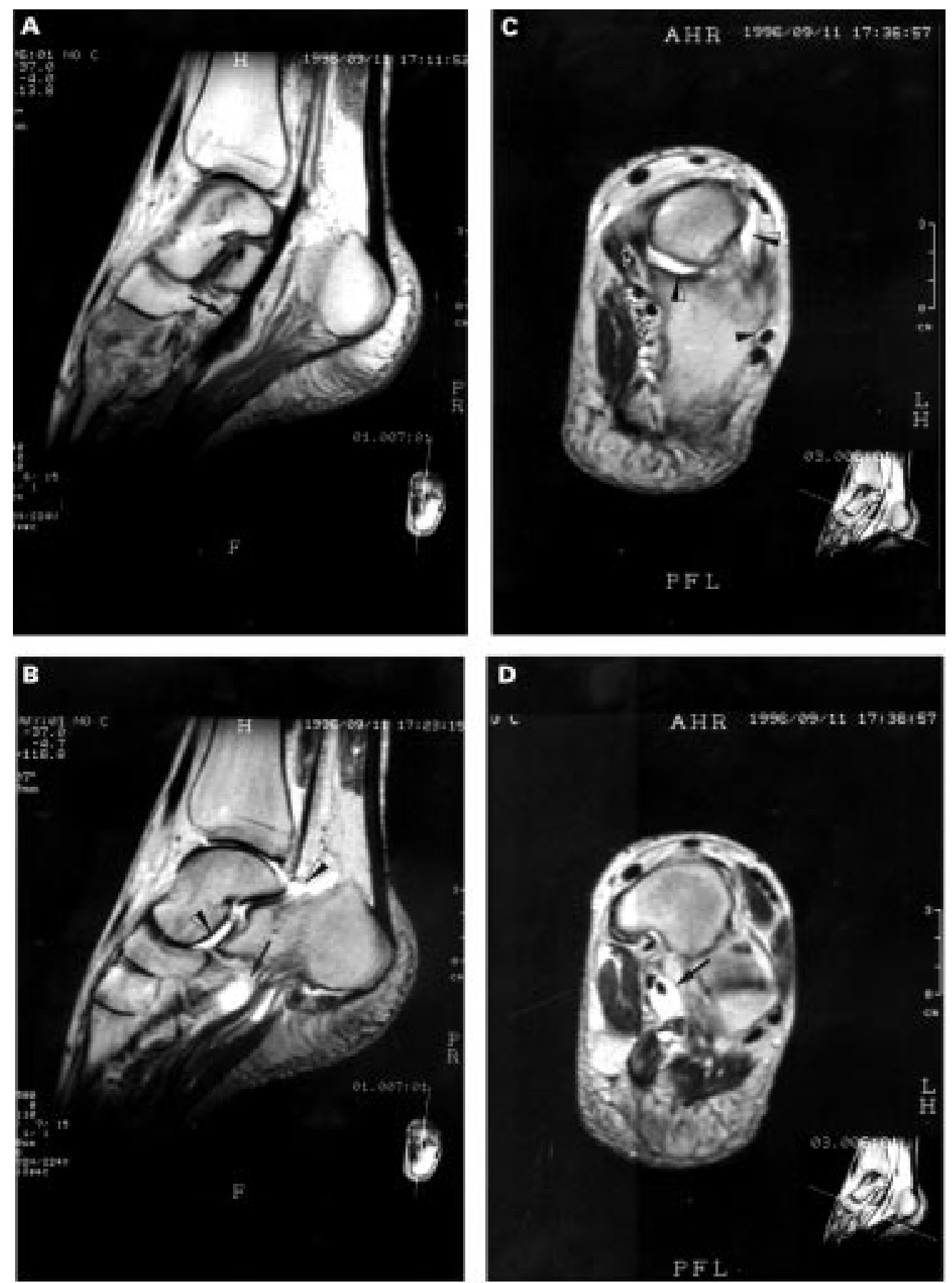

Figure 2 MRI of the foot of RS3PE syndrome. (A) Sagittal T1 weighted section shows marked tenosynovitis of tibialis anterior tendon (black arrow). (B) Sagittal T2 weighted section shows marked tenosynovitis of tibialis anterior tendon (black arrow), ankle joint effusion extending into Kager's triangle (black/white arrow) and talonavicular joint effusion (black/white arrow). (C) Coronal T2 weighted section at the apex of the talus shows talonavicular joint effusion (black/white arrow), tenosynovitis of flexor hallucis longus tendon (double open arrowhead), tenosynovitis of flexor digitorum longus tendon (open arrowhead), tenosynovitis of tibialis posterior tendon (open white arrow) and tenosynovitis of peroneal tendons (black arrowhead). (D) Coronal T2 weighted section through the navicular bone shows marked tenosynovitis of tibialis anterior tendon (black arrow).

negative for rheumatoid factor (Rose-Waaler titre $\leqslant 1: 40$ or nephelometric determination $\leqslant 20 \mathrm{IU} / \mathrm{ml}$ on two or more occasions) and fluorescent antinuclear antibodies (titre $\leqslant 1: 32$ ). At the end of the follow up period all 23 patients had withdrawn from treatment.

Twenty patients had a single episode and the other three had a second episode during the follow up. At diagnosis 13 episodes (56\%) were symmetric and 10 unilateral $(44 \%)$. The hands and wrists were involved in 17 episodes $(74 \%)$, feet in two episodes (9\%) and both hands and feet in four episodes (17\%). In the three episodes of relapse the oedema involved hands. The swelling and pitting oedema were most prominent over the dorsum of the hands and wrists, the ankles, and the tops of the feet. At inspection swelling and tenderness were localised, especially along the course of hand extensor tendons in 21 of 23 patients (91\%), flexor tendons of the hand in nine of $23(39 \%)$, foot estensor tendons in six of $23(26 \%)$, peroneal 
tendons in three of $23(13 \%)$, posterior tibial tendons in three of $23(13 \%)$. Given the difficulty of clinical detection of joint synovitis because of the marked oedema, wrist synovitis could be identified in 19 of 23 patients (83\%). In five of 23 patients (22\%) a concomitant knee arthritis was present. In two of these patients aspiration of synovial fluid was possible and examination showed $4.0 \times 10^{9} / 1$ white blood cells in the first case and $5.2 \times 10^{9} / 1$ in the second, with neutrophils $52 \%$ and $68 \%$ at differential count, respectively. CTS occurred in five of 23 patients $(22 \%)$.

The radiographs showed no evidence of articular erosions, typical calcifications of chondrocalcinosis, and/or sacroiliitis.

Nineteen patients were treated with prednisone and four with non-steroidal antiinflammatory drugs (NSAIDs). The swelling and oedema promptly remitted (median: one week, range: 1-3 weeks) in all corticosteroid treated patients, while the response to NSAIDs was more gradual (median: 3 weeks, range: $2-4$ weeks).

Residual mild contractures were observed in two of 23 patients (9\%). The first patients had a mild limitation of dorsal and palmar flexion of the right wrist (range of motion reduced by $5-10$ degrees in both movements) with thickening of wrist extensor and flexor tendons, the second had a mild flexion contracture of fingers with flexor thickening. None of the 23 patients developed articular erosions on hand and/or foot radiographs at the end of the follow up.

ESR was normal at diagnosis in three of 23 patients $(13 \%)$, while CRP was normal in one of $23(4 \%)$. One patient had both normal ESR and CRP values at diagnosis. HLA-B7 was present in two of 13 patients and 14 of 126 controls $(15 \%$ versus $11 \%, \mathrm{p}=\mathrm{NS})$. No significant association with other class I HLA antigens was observed.

HAND AND FOOT MRI FINDINGS

Hand MRI was performed in five patients and foot MRI in two. Tenosynovitis (defined by the presence of fluid in the tenosynovial sheaths) of hand extensor tendons was observed in three patients, flexor hand tenosynovitis in one patient and hand joint synovitis in two patients (fig 1). Tenosynovitis of peroneal tendons was observed in both the patients studied with MRI; one patient also associated tibialis tendons, flexor hallucis longus tendon and flexor digitorum longus tendon tenosynovitis and foot joint synovitis (fig 2). None of the five healthy controls showed evidence of fluid collection in hand flexor or extensor tenosynovial sheaths

COMPARISONS BETWEEN THE PATIENTS WITH PURE RS3PE SYNDROME AND PMR WITH AND WITHOUT DISTAL EXTREMITY SWELLING WITH PITTING OEDEMA

The results of these comparisons are reported in table 1. A trend of higher frequency in men was observed for pure RS3PE compared with PMR without distal extremity swelling with pitting oedema $(p=0.06)$. The distribution of the patients in 10 year age groups was similar in the three conditions. No patients under 50 were observed, the disease frequency increased significantly with age and the highest frequency was present in the age group 70-79 years.

Systemic signs or symptoms, or both, were significantly less frequent in pure RS3PE syndrome compared with PMR with or without distal extremity swelling with pitting oedema. The duration of disease before diagnosis, the duration of prednisone treatment, and the cumulative prednisone dose were significantly lower in pure RS3PE patients compared with the other two groups. The duration of follow up was significantly shorter in pure RS3PE patients compared with patients with PMR without distal oedema. The initial prednisone dose was significantly lower in pure RS3PE syndrome compared with the patients with PMR and distal oedema. Relapse/ recurrences were significantly less frequent in pure RS3PE patients compared with PMR with distal oedema, while a trend of reduced frequency in comparison with PMR without distal oedema $(p=0.08)$ was observed. Residual mild contractures were significantly more frequent in pure RS3PE syndrome compared with PMR patients without distal oedema.

\section{Discussion}

In our prospective follow up study we examined the clinical features of 23 patients with isolated RS3PE syndrome. Unlike the study by $\mathrm{McCa}-$ rty, we included patients with unilateral or lower extremity involvement, or both. Those authors empirically selected only patients with symmetrical upper limb involvement, while the spectrum of this condition also includes patients with unilateral findings. ${ }^{3}{ }^{4}$ In our patients the clinical examination revealed an extensor tenosynovitis of the hands and feet as the predominant lesions, while hand flexor, posterior tibial and peroneal tenosynovitis were less frequently observed. Hand and foot MRI confirmed that the inflammation of the tenosynovial sheaths is the hallmark of this condition and hand extensor tendon apparatus is the anatomical structure more frequently involved. Joint synovitis was more difficult to appreciate at clinical examination because of the impressive swelling and oedema involving the hands and feet. However, MRI demonstrated a concomitant joint synovitis in some patients.

In our series we observed a very low frequency of residual hand contractures and tendon thickening. Only $9 \%$ of our patients presented this complication in a mild form compared with $61 \%$ observed by McCarty et l. $^{1{ }^{2}}$ In contrast with the series of McCarty, the inclusion of patients with unilateral RS3PE and the prospective design of our study may have contributed to evaluate less severe form of the disease and to explain the low frequency of residual hand contractures observed in our series.

Furthermore, our study did not confirm the association of RS3PE syndrome with HLA-B7 in Italian patients. Our findings confirm the frequent association with knee synovitis and CTS. As in previous descriptions the clinical features rapidly responded to corticosteroids and rarely recurred. 
Many similarities exist between RS3PE syndrome and PMR. In addition, a percentage of PMR patients oscillating between 8-12\% presents distal extremity swelling with pitting oedema similar to that observed in RS3PE. ${ }^{5}$ These distal findings were part of PMR inflammatory processes (concurrence with proximal PMR manifestations, age at onset > 50 years, prompt response to corticosteroids), whose clinical spectrum may be wider than what has been believed, including patients with RS3PE syndrome. Our long term, prospective follow up study on PMR and pure RS3PE syndrome have provided a unique opportunity to evaluate the relation between these two conditions. We separately compared patients with pure RS3PE syndrome and PMR with or without distal extremity swelling with pitting oedema. Even though there was a predominance of men in pure RS3PE syndrome and of women in PMR, the difference was not significant. The elderly age of disease onset, the absence of rheumatoid factor, the increased acute phase reactants, the similar frequency of peripheral synovitis and CTS, the rapid response to corticosteroids are common characteristics of both PMR and RS3PE syndrome. Furthermore, the distribution of the patients in 10 year age groups was similar in the two conditions. No patients under 50 were observed, the disease frequency increased significantly with age and the highest frequency was present in the age group 70-79 years.

Similar characteristics were also present in a group of patients who had clinical features of overlap between the two syndromes. Furthermore, no differences in the frequency of HLA class I antigens, in particular of HLA-B7, were observed in the three groups of patients. The concurrence of the two syndromes and their similarities suggest they might be manifestations of the same disease. The group of patients with PMR and pitting oedema may represent the ring of conjunction between the more frequently encountered patients with only proximal symptoms and the less frequent patients with prominent distal manifestations. Some of the patients with pitting oedema (with or without PMR proximal symptoms) would meet the ACR criteria for $\mathrm{RA}^{17}$ at diagnosis. However, these distal manifestations promptly remitted without developing erosions and none of our patients experienced a course of prolonged and persistent synovitis. Their articular disease is completely different from classic erosive RA and seems to be part of the inflammatory processes of PMR. The presence of distal swelling with oedema seems to characterise a more benign condition with shorter duration of treatment, lower cumulative corticosteroid dose and lower frequency of systemic signs/symptoms and relapse/ recurrence.

We did not observe patients with biopsy confirmed GCA in either the PMR with distal oedema group or the pure RS3PE group. However, the differences compared with the frequency of biopsy confrimed GCA in patients with PMR without distal pitting oedema were not significant. A higher number of patients with distal oedema need to be enrolled to evaluate if a reduced association with GCA is really present in this group of patients.

The similarities of MRI findings in PMR with distal pitting oedema ${ }^{21} 22$ and pure RS3PE seem to indicate that the involvement of extraarticular synovial structures represents the common anatomical target of the inflammatory process in these two conditions.

In conclusion, our study confirms the presence of patients with pure RS3PE syndrome. The distal extremity oedema occurs more frequently in a symmetric pattern, predominantly in upper limbs, even if unilateral or isolated lower extremity involvement may also occurr. MRI findings confirm the clinical impression of a predominant tenosynovial involvement. Flexor contractures were rarely observed and mild. No association of pure RS3PE syndrome with HLA-B7 was present in Italian patients.

The clinical, demographic, and MRI findings observed in patients with RS3PE syndrome suggest some similarities with those of PMR patients.

1 McCarty DJ, O'Duffy DJ, Pearson L, Hunter JB. Remitting seronegative symmetrical synovitis with pitting edema. JAMA 1985;254:2763-7.

2 Russell EB, Hunter JB, Pearson L, McCarty DJ. Remitting, seronegative, symmetrical synovitis with pitting edema - 13 additional cases. J Rheumatol 1990;17 633-9.

3 Parisier KM, Canoso JJ. Remitting, seronegative (A)symmetrical synovitis with pitting edema - Two cases of RS3PE syndrome. J Rheumatol 1991;18:1260-2.

4 Olivieri I, Padula A, Favaro L, Oranges GS, Ferri S. RS3PE syndrome with unilateral involvement. [Letter]. J Rheumaol 1994;21:372.

5 Salvarani C, Gabriel S, Hunder GG. Distal extremity swelling with pitting edema in polymyalgia rheumatica. Arthritis Rheum 1996;39:73-80.

6 Salvarani C, Cantini F, Macchioni L, Olivieri I, Niccoli L, Padula A, et al. Distal musculoskeletal manifestations in polymyalgia rheumatica: a prospective follow-up study. Arthritis Rheum, 1998;41:1221-6.

7 Olivieri I, Padula A, Pierro A, Favaro L, Oranges GS, Ferri . Late onset undifferentiated seronegative spondylarthropathy. J Rheumatol 1995;22:899-903.

8 Olivieri I, Padula A, Favaro L, Pierro A, Oranges GS, Ferri S. Dactylitis with pitting oedema of the hand in longstanding ankylosing spondylitis. Clin Rheumatol 1995;14:701-4.

9 Schaeverbeke T, Fatout E, Marcé S, Vernhes J-P, Hallé O, Antoine J-F, et al. Remitting seronegative symmetrical synovitis with pitting oedema: disease or syndrome? Ann synovitis with pitting oedema:
Rheum Dis $1995 ; 54: 681-4$.

10 Olivieri I, Salvarani C, Cantini F. Remitting distal extremity swelling with pitting edema: a distinct syndrome or a clinical feature of different inflammatory rheumatic diseases? J Rheumatol 1997;24:249-52.

11 Bhakta BB, Pease CT. Late-onset rheumatoid arthritis: is pitting oedema of the hands at onset a good prognostic indicator? Br J Rheumatol 1997;36:214-19.

12 Cantini F, Niccoli L, Olivieri I, Barozzi L, Pavlica P, Bozza A, et al. Remitting distal lower extremity swelling with pitting oedema in acute sarcoidosis. [Letter]. Ann Rheum Dis 1997;56:565-6.

13 Olivo D, Mattace R. Concurrence of benign edematous polysynovitis in the elderly (RS3PE syndrome) and endome-

4 Tada Y, Sato H, Yoshizawa S, Kimura H, Kitamura M, Kusabe $\mathrm{T}$, et al. Remitting seronegtive symmetrical synovitis with pitting edema associated with gastric carcinoma. J Rheumatol 1997;24:974-5.

15 Cantini F, Olivieri I, Salvarani C. More on RS3PE as paraneoplastic syndrome. [Letter]. J Rheumatol 1998;25: $188-9$

16 Healey LA. Long-term follow-up of polymyalgia rheumatica: evidence for synovitis. Semin Arthritis Rheum 1984;13:322-8

17 Arnett FC, Edworthy SM, Bloch DA, McShane DJ, Fries $\mathrm{JF}$, Cooper NS, et al. The American Rheumatism Association 1987 revised criteria for the classification of rheumatoid arthritis. Arthritis Rheum 1988;31:315-24.

18 Dougados $M$, van der Linden S, Juhlin R, Huitfeldt B, Amor B, Calin A, et al. The European spondylarthropathy study group preliminary criteria for the classification of spondylgroup preliminary criteria for the classification of

19 Polley HF, Hunder GG. Rheumatologic interviewing and physical examination of the joints. 2nd ed. Philadelphia: WB Saunders, 1978 
20 Terasaki P, McClelland JD. Microdroplet assay of human serum cytotoxins. Nature 1964;204:998-1000

21 Salvarani C Cantini F, Olivieri I, Barozzi L Macchioni PL Niccoli L, et al. Proximal bursitis in active polymyalgia heumatica. Ann Intern Med 1997;127:27-31.
22 Olivieri I, Salvarani C, Cantini F, Barozzi L, Pavlica P, Macchioni PL. Distal extremity swelling with pitting edema in polymyalgia rheumatica: a case studied with magnetic resonance imaging. [Letter]. Clin Exp Rheumatol 1997;15: $710-11$.

\section{Historical images}

Series editors: W Grassi, C Cervini
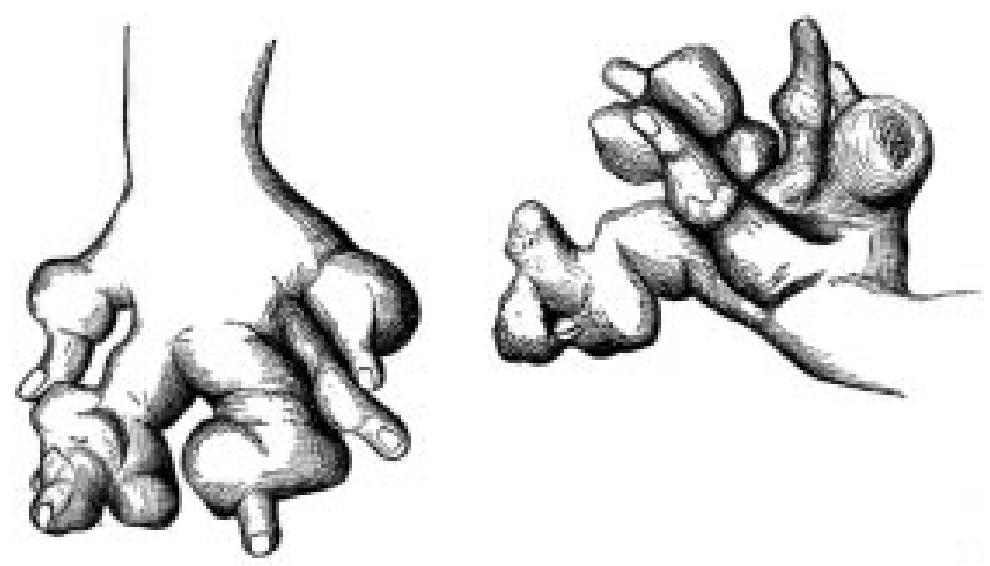

Figure 14 Hyperostosis of the fingers in a 20 year old woman. Kirmisson. Malattie degli arti. In: Duplay S, Reclus P, eds. Trattato di chirurgia. Turin: Unione Tipografico Editrice, 1895. 